\title{
Design And Experimental Validation Of A Lateral LPV Control of Autonomous Vehicles*
}

\author{
H. Atoui ${ }^{1,2}$, V. Milanés ${ }^{1}$, O. Sename ${ }^{2}$ and John J. Martinez ${ }^{2}$
}

\begin{abstract}
This paper presents a multi-scenario full-range speed lateral automated vehicle controller. A speed-dependent LPV model is designed to deal with two different situations: 1) vehicle tracking capabilities to follow a pre-defined trajectory; and 2) vehicle response to sudden reference changes as occur either when activating the automated system for the first time or when performing a lane-change. The proposed solution is based on the Linear Parameter Varying (LPV) control approach, where an output-feedback dynamical controller is designed based on the Linear Matrix Inequalities (LMIs). The control synthesis is carried out using the Linear Fractional Transformation approach, to reduce the conservatism, combined with the $\mathcal{H}_{\infty}$ control problem. Simulation results show the tracking performance and the smoothness of the control inputs which provides a comfortable riding. Finally, the algorithm has been implemented on a robotized Renault ZOE and validates on test tracks, providing encouraging results.
\end{abstract}

\section{INTRODUCTION AND MOTIVATION}

Autonomous vehicles domain remain as a hot research topic because of its potential benefits. Among them, it reduces traffic flow, accidents, fuel consumption and also it allows anyone to travel by car regardless of his abilities. Assuming negligible vertical motion of the vehicle, the control consists of the longitudinal and the lateral controls only. The longitudinal control is mainly used to ensure the safe distance between the vehicles by regulating the automated vehicle speed. Let us mention, among others, [1], where Cooperative Adaptive Cruise Control (CACC) is implemented using Youla-Kucera parameterization (YK parameterization) to control the longitudinal dynamics in critical situations. Thus, this control concerns the throttle and the brake actuators of the vehicle. On the other hand, the lateral control involves the vehicle's steering to track a position-planning reference, ideally providing safety and a comfortable riding.

Vehicle lateral control is an attractive problem in the domains of Intelligent Transportation Systems (ITS) and in Automotive Control. The major roles of this control is to deal with the lane keeping, turning, lane changing and avoidance maneuvers. As shown in the literature, the lateral control is divided into two main components ([2], [3]): 1) a feedforward term which is related to the reference trajectory

\footnotetext{
*Institute of Engineering Univ. Grenoble Alpes

1 H. Atoui and V. Milanés are with the Research Department, Renault SAS, 1 Avenue de Golf, 78280 Guyancourt, France hussam.atoui, vicente.milaneserenault.com

${ }^{2} \mathrm{H}$. AtouiO. Sename and John J. Martinez are with GIPSAlab, CNRS, Univ. Grenoble Alpes, Grenoble INP, 38000 Grenoble, France. hussam. Atouidetu.univ-grenoble-alpes.fr, olivier.sename@grenoble-inp.fr,

john-jairo.martinez-molina@grenoble-inp. fr
}

by considering the road curvature. The reference curvature is chosen to be away by a look-ahead distance which varies with respect to the vehicle's speed, in order to achieve smooth steering action; 2) the feedback compensator that minimizes the current vehicle errors to stabilize the lateral position.

\section{A. Related Works}

This section presents a review of different control techniques applied to the lateral automated vehicle control problem. In 1996, a steering controller was designed to track the current lane center for both curved and straight lanes regardless of the radius of curvature of the road [4]. In [5], the road curvature was used as a feedforward term, and a PID controller was applied on a weighted sum of the lateral offset and the heading angle. Other techniques were released for controlling steering, such as $\mathcal{H}_{\infty}$, Adaptive, and PID, which were compared in [6].

To maintain human steering behavior, the look-ahead systems were used by measuring the lateral displacements from the reference at a distance in front of the vehicle. Such systems mainly use the Global Positioning System (GPS), where a high integrity navigation system of an automated vehicle, based on the fusion between the Inertial Measurement Unit (IMU) and the GPS, is developed and implemented in [7]. This allows to predict the future lateral error and try to minimize it smoothly as the human driver does. In [3], a feedforward term was added to the feedback lateral controller as an anticipatory that is common in the human drivers. In addition, the computer vision algorithm was used also to measure the lateral error and the heading error (between the vehicle and the lane center) at an adjusted look-ahead distance from the vehicle as in [8]. The authors showed the role of look-ahead system with respect to the delay of the vision processing and how it is useful to be adjusted according to the vehicle's longitudinal speed and the road curvature. In [2], it was observed that the steering rate is proportional to the combination heading/lateral error, according to human driver's data analyzed from a double lane change. In this approach, the poles of the controller varies with respect to the speed and the look-ahead distance. Thus, it was applied by fixing both parameters as a Linear Time-Invariant controller (LTI).

For more development, the authors in [9] have designed a multi-task controller (lane tracking and lane changing), considering two controllers with a fixed-constant speed but different look-ahead distances. As a result, they applied a transition between both controllers (depending on the lateral 
error) using Youla-Kucera (YK) parametrization. Simulation and experimental validation where done for a constant speed and interesting results were obtained. From this point of view, it was obvious to search for a Linear Parameter-Varying approach which provides a full range of speed controller. The main advantage of this approach is that it precisely models any nonlinear model as a set of LTI models that permits the usage of linear control design tools.

Several LPV approaches were used in previous works. In [10], the authors used LMI-based LPV-LQR/H $\mathcal{H}_{2}$ cascaded controllers using the polytopic LPV approach. The same approach was also used to design an LPV-MPC controller for a racing car as shown in [11]. In [12], the authors used the LPV gridded-based approach to enhance the vehicle rollover stability, where a braking action is taken in critical situations. In this paper, the Linear Fractional Transformation (LPV/LFT) $\mathcal{H}_{\infty}$ control approach is discussed. This approach is well shown in [13] concerning continuous and discrete-time LFT/H $\mathcal{H}_{\infty}$ control design and it was used in several applications ([14], [15]).

\section{B. Paper Contributions}

The main contribution of this paper is to design a single full speed-range controller which can deal, comfortably, with both lane-change and lane-tracking problems. This can be summarized as follows:

- The nonlinear bicycle model is approximated as an LFT model to cover the range of velocities.

- An LMI-based LPV/H $\mathcal{H}_{\infty}$ control is applied to provide noise/disturbance rejection, to limit the control inputs according to the real steering actuator and to achieve smooth steering riding.

- The designed controller is independent on the lookahead system, where the look-ahead time will be adjusted during generating the tracking reference as a function of the lateral error and speed. As a result, the look-ahead time is changing to handle large lateral errors (lane changing) and the small ones (lane tracking).

- Simulation and implementation results are represented to ensure the performance of the controller concerning tracking, actuator limitations and noise/disturbance rejection.

- For the first time, the LPV/LFT approach is implemented on an automated vehicle and the results are shown at the end.

This paper is organized as follows. In Section 2, a brief theoretical explanation on the LPV formulation structure and the control design of LPV/H $\mathcal{H}_{\infty}$. The control scheme of a full speed-range controller with multiple tasks (lane change and path tracking) is shown in Section 3. Section 4 presents the simulation results obtained by the designed controller and the demanded performances are analysed. In section 5, experimental validation is demonstrated to ensure the reliability of the work done. Finally, section 6 collects some conclusions and remarks.

\section{MODEL FORMULATION}

This section focuses on vehicle modelling for control. The main dynamics of the bicycle model are the longitudinal and the lateral dynamics.

\section{A. Lateral Dynamic Model}

For the lateral control, only the lateral dynamics are extracted. Referring to [16] and [17], the lateral dynamic model is derived below.

$$
\left\{\begin{array}{l}
\dot{v}_{y}=\frac{F_{y f} \cos \delta+F_{y r}}{m}-w v_{x} \\
\dot{w}=\frac{F_{y f} l_{f} \cos \delta-F_{y r} l_{r}}{I},
\end{array}\right.
$$

where $v_{x}, v_{y}$ and $w$ are the longitudinal, lateral and rotational velocities in the vehicle's frame, respectively. $\delta$ is the control input, the steering angle of the front tire. $F_{y f}$ and $F_{y r}$ are the lateral forces applied to the front and rear tires, respectively. $I, m, l_{f}$ and $l_{r}$ are the vehicle's inertia, mass and the distance from the center of gravity to the front and rear wheel axes respectively.

The lateral forces are approximated to be:

$$
\begin{gathered}
F_{y f}=C_{f}\left(\delta-\frac{v_{y}}{v_{x}}-\frac{l_{f} w}{v_{x}}\right), \\
F_{y r}=C_{r}\left(-\frac{v_{y}}{v_{x}}+\frac{l_{r} w}{v_{x}}\right),
\end{gathered}
$$

where $C_{f}$ and $C_{r}$ represent the stiffness of the front and rear wheel-tires.

\section{B. LPV Model Formulation}

Considering a small steering angle $\delta$, observe the following LPV state-space representation, considering $\rho(t)=v_{x}$ :

$$
G(\rho)\left\{\begin{aligned}
\dot{x}(t) & =A(\rho) x(t)+B(\rho) u(t) \\
y(t) & =C x(t)+D u(t)
\end{aligned}\right.
$$

where:

$$
\begin{aligned}
& x(t)=\left[\begin{array}{l}
v_{y} \\
w
\end{array}\right], u(t)=\delta, B(\rho)=\left[\begin{array}{c}
\frac{1}{m} C_{f} \\
\frac{1}{m} C_{f} l_{f}
\end{array}\right], \\
& A(\rho)=\left[\begin{array}{cc}
-\frac{C_{r}+C_{f}}{m v_{x}} & -\frac{C_{f} l_{f}-C_{r} l_{r}}{m v_{x}}-v_{x} \\
-\frac{C_{f} l_{l_{x}}-l_{r} C_{r}}{I v_{x}} & -\frac{C_{f} l_{f}+l_{r}^{2} C_{r}}{I v_{x}}
\end{array}\right],
\end{aligned}
$$

The longitudinal speed to be bounded as:

$$
v_{x} \in[3,30] \mathrm{m} / \mathrm{s}
$$

As shown in Fig 2, the vehicle model is completed by an actuator model (first order transfer function + input delay) $\left(G_{a c t}\right)$ for the control design part.

The LPV/LFT model of a system is defined as a lower or upper Linear Fractional Representation (LFR) between the known LTI model (parameter-invariant) and the varyingparameters block as shown in Fig. 1. This model was presented in [18], where it was applied on an inverted pendulum. Moreover, it was used in the control of autonomous underwater vehicles in [14]. The aim was to design a gainscheduling controller with ensuring a performance adaptation when the sampling interval varies. In [15], a discrete-time $\mathrm{LPV} / \mathrm{LFT} \mathcal{H}_{\infty}$ controller for a diesel engine common rail 


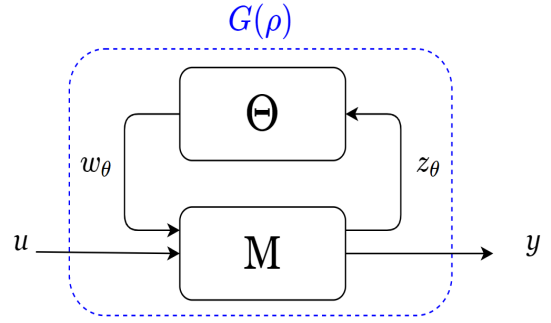

Fig. 1: Linear fractional representation of a parametervarying model

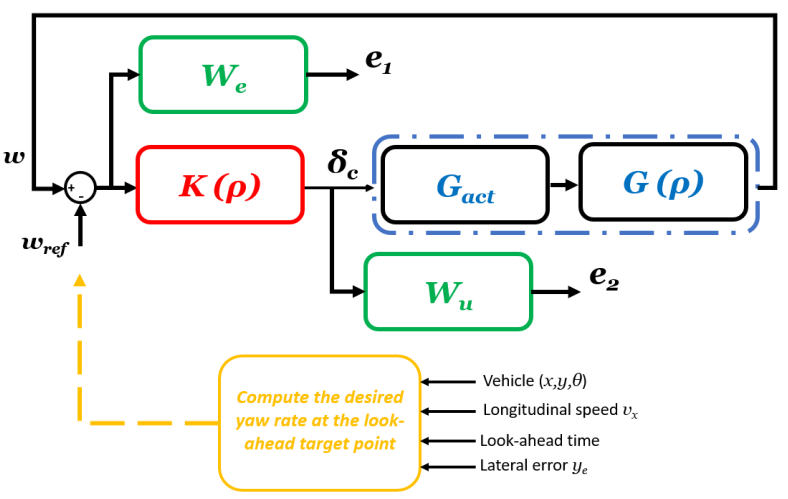

Fig. 2: Control scheme

injection systems, real implementation was done also. At each instant, the parameters in the block are updated and then injected as an input to the known LTI model (M) to ensure the scheduling with respect to the parameters' variations. The upper LFT interconnection of the model can be written as:

$$
\begin{gathered}
{\left[\begin{array}{c}
\dot{x} \\
z_{\theta} \\
y
\end{array}\right]=\left[\begin{array}{ccc}
A & B_{\theta} & B \\
C_{\theta} & D_{\theta \theta} & D_{\theta 1} \\
C & D_{1 \theta} & D
\end{array}\right]\left[\begin{array}{c}
x \\
w_{\theta} \\
u
\end{array}\right]} \\
w_{\theta}=\theta z_{\theta}
\end{gathered}
$$

where $\Theta$ is the time-varying operator block and is introduced as:

$$
\Theta=\operatorname{blockdiag}\left(\theta_{1} I_{r_{1}}, \ldots, \theta_{k} I_{r_{k}}\right)
$$

where $r_{i}>1$ presents the number of occurrences of the varying-parameter $\theta_{i}$.

\section{LPV/H $\mathcal{H}_{\infty}$ CONTROL DESIGN}

The LPV/robust control approach is well known in the literature and has been used in several applications, as seen in [19] and in [20]. This paper is concerned with the design of an $\mathrm{LPV} / \mathcal{H}_{\infty}$ controller $\mathrm{K}(\rho)$ following the control scheme represented in Fig. 2. Notice that the yaw rate reference $w_{\text {ref }}=v_{x} \times k$, where $k$ is the road curvature. Moreover, knowing that the curvature is written as a function of the look-ahead time (see [2]), a smooth or aggressive maneuver reference can be achieved by changing the look-ahead time.

First of all, let us choose the required performances using frequency domain weighting functions. Usually, two

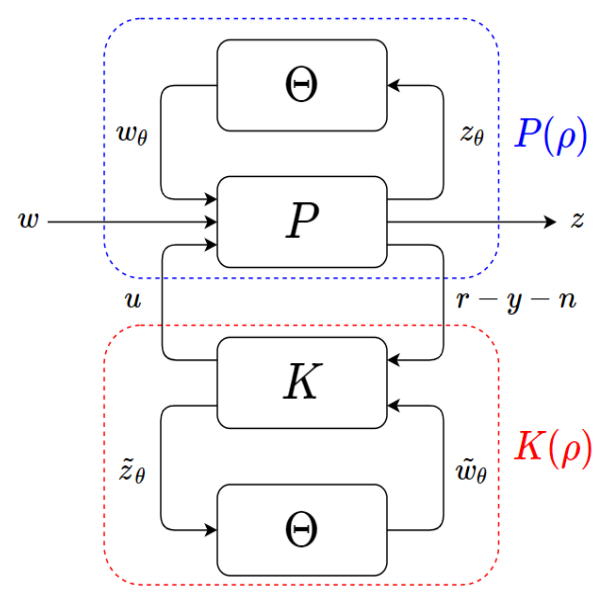

Fig. 3: General control configuration

weighting functions are used to minimize both the tracking error and the control effort according to the actuator limits (see Fig. 2). The signals in $y$ are used to be available measurements in vehicles by making use of sensors like GPS, IMU and wheel encoders.

The main objective of the $\mathcal{H}_{\infty}$ control is to minimize the $\mathcal{L}_{2}$ induced gain from the input $r$ to the controlled output $\mathrm{z}=\left[\begin{array}{ll}e_{1} & e_{2}\end{array}\right]^{T}$.

\section{A. Tracking specification (using $W_{e}$ )}

The chosen weighting transfer function is of the form:

$$
W_{e}(s)=\frac{\frac{s}{M_{s}}+w_{b}}{s+w_{b} \epsilon}
$$

where the parameters $M_{s}, w_{b}$ and $\epsilon$ are tuned as follows:

- $M_{s}=2$, to ensure robustness at any frequency.

- $w_{b} \geq 10$, to get fast tracking (short rise-time).

- $\epsilon \leq 10^{-4}$, it represents the steady-state tracking error.

\section{B. Specification on the control input limitations ( $\left.W_{u}\right)$}

Additionally, a filter in included for the steering control input $\delta$ to minimize the actuator control effort. The filter has the following form:

$$
W_{u}(s)=\frac{s+\frac{w_{b_{u}}}{M_{u}}}{\epsilon_{u} s+w_{b_{u}}}
$$

The parameters $M_{u}, w_{b_{u}}$ and $\epsilon_{u}$ are chosen as follows:

- $M_{u}$ represents the limitations on the maximum allowed effort of the actuators.

- $w_{b_{u}}$, is related to the actuator bandwidth.

- $\epsilon_{u} \leq 10^{-2}$, is concerned with the noise rejection from the control inputs at high frequencies.

\section{Generalized Plant}

The generalized plant $P(\rho)$ is structured to consider the chosen weights in addition to the LPV model. The state vector of $P(\rho)$ is $x_{P}=\left[\begin{array}{llll}x & x_{a c t} & x_{W_{e}} & x_{W_{u}}\end{array}\right]^{T}$, and the controlled output $\mathrm{z}=\left[\begin{array}{ll}e_{1} & e_{2}\end{array}\right]^{T}$ represents the objective function to be optimized in the control design problem. The 


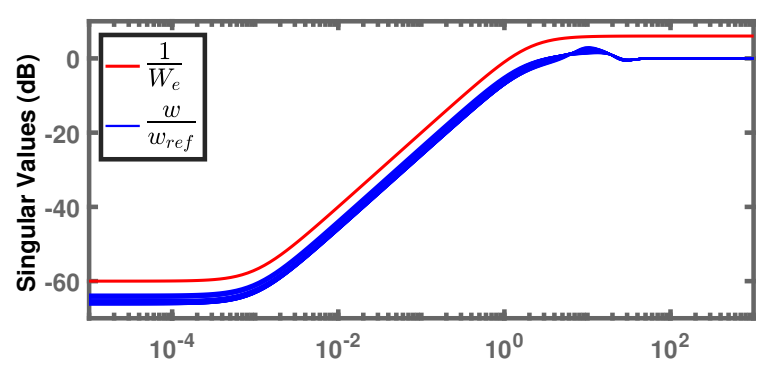

Fig. 4: Sensitivity Functions $S$

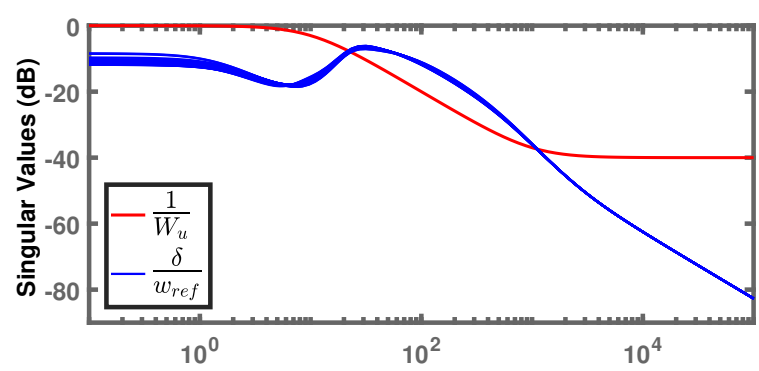

Fig. 5: Sensitivity Functions KS

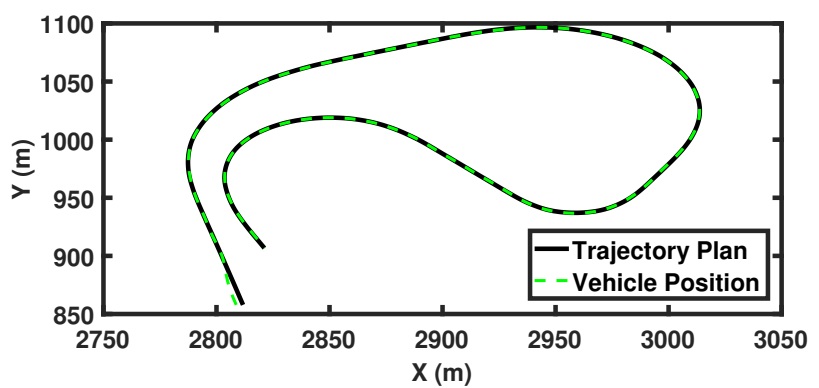

Fig. 6: Planned and Controlled Trajectories

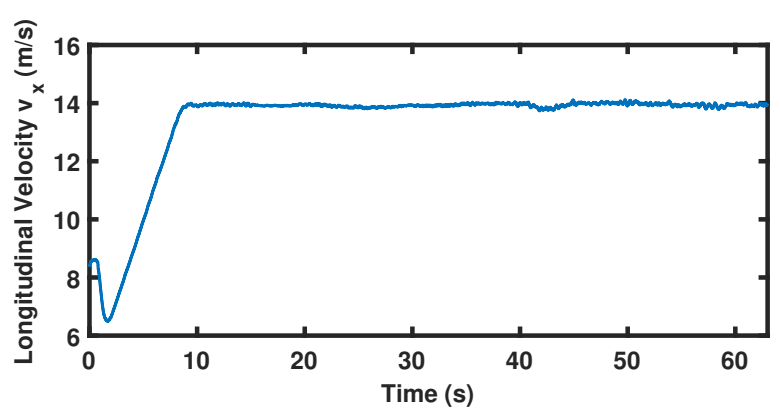

Fig. 7: Longitudinal speed $v_{x}(\mathrm{~m})$

\section{E. Frequency Domain Analysis}

To analyse the results, this paragraph discusses if the controller satisfies the requirements according to the designed weights $W_{e}$ and $W_{u}$. From Fig. 2, the used sensitivity functions are computed as:

$$
\begin{aligned}
\frac{w_{e}}{w_{r e f}} & =\frac{w_{r e f}-w}{w_{r e f}}=1-\frac{w}{w_{r e f}}=\frac{1}{1-G(\rho) K(\rho)}, \\
\frac{\delta}{w_{r e f}} & =\frac{\delta}{w_{e}} \times \frac{w_{e}}{w_{r e f}}=\frac{K(\rho)}{1-G(\rho) K(\rho)},
\end{aligned}
$$

It is worth to mention that each plot of the sensitivity functions corresponds to a frozen value of the varyingparameter $v_{x}$ at an instant of time. Regarding Fig. 4 shows that all the closed-loop systems are respecting the steadystate tracking error (at $w=0$ ), and the robust margin $\left(\max _{\forall w}\left\|\frac{r-y}{r}\right\|<6 d B\right)$. Fig. 5 shows that the control input $\delta$ is respecting somehow the maximum limit and the demanded band-width. Therefore, the controller perfectly achieved the demanded tracking and control-effort performances. The next subsection will discuss the results obtained after the simulation done in discrete-time domain.

\section{SIMULATION RESULTS}

Simulations were done using the vehicle nonlinear model (eqs. (1) and (2)) in discrete-time domain with a sampling time $T_{s}=10 \mathrm{~ms}$. To test the lateral control performance, a chosen scenario with four successive turns is used as can be seen in Fig. 6. Moreover, to approve the controller capabilities in large lateral errors, the lateral error is initialized to be large enough. The velocity was selected to increase initially reaching $v_{x}=14 \mathrm{~m} / \mathrm{s}$ and then staying constant for a range

where $K_{11}, K_{12}, K_{21}$ and $K_{22}$ are obtained from the LMI's. 


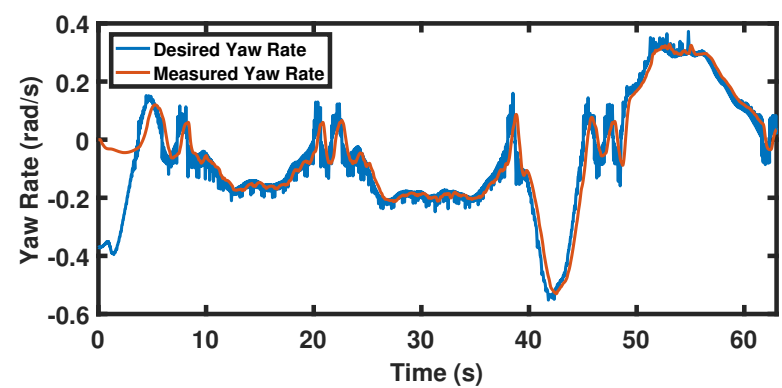

Fig. 8: Desired and measured yaw rates ( $\mathrm{rad} / \mathrm{s})$

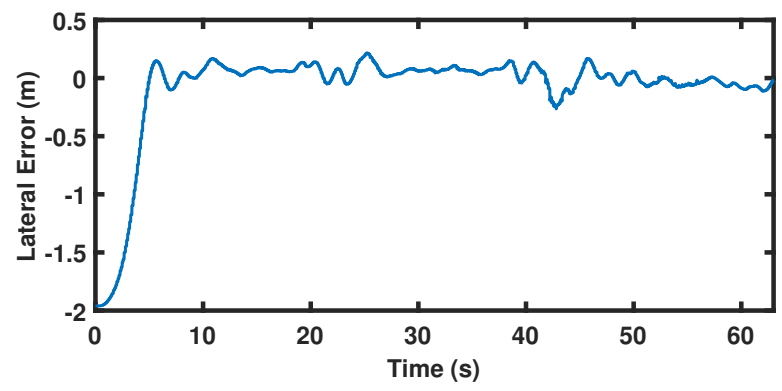

Fig. 9: Lateral error (m)

of time (see Fig. 7). Notice that this velocity was taken from a real data measurement.

In Fig. 8, the tracking performance is shown with a noisy reference $w_{r e f}$. As observed before in the frequency-domain analysis, the controller doesn't have a fast rising response, however the tracking-error is very small at the steady-state. Fig. 9 shows an acceptable lateral error (|lateral error $\mid<$ $0.5 \mathrm{~m}$ ) of the vehicle along time. It is worth to shed light on how the lateral error was decreased smoothly after the injected initial condition, thanks to the strategy used which changes the look-ahead time with respect to the lateral error. Notice that the main objective of the controller is to minimize the lateral error with a smooth steering actions. Thus, the acceptability of such errors came with the fact of ensuring a smooth and comfortable riding which can be clearly analyzed from the smoothness of the steering angle shown in Fig. 10. Also, the limitation on the control input was satisfied regarding the maximum absolute value reached around 0.11 rad.

After confirming that the simulations satisfy the demanded performance, the next section will discuss the implementation of the designed controller on a real vehicle.

\section{REAL IMPLEMENTATION}

The designed controller was tested on a robotized electric Renault ZOE which is prepared for lateral and longitudinal controls. To the best of our knowledge, former LPV control designs for automated vehicle lateral control have not been implemented on real daily vehicles, which generates the curiosity to evaluate its real performance.

The test was done in the test-track of Satory shown in Fig. 11. This test-track is challenging concerning the bad

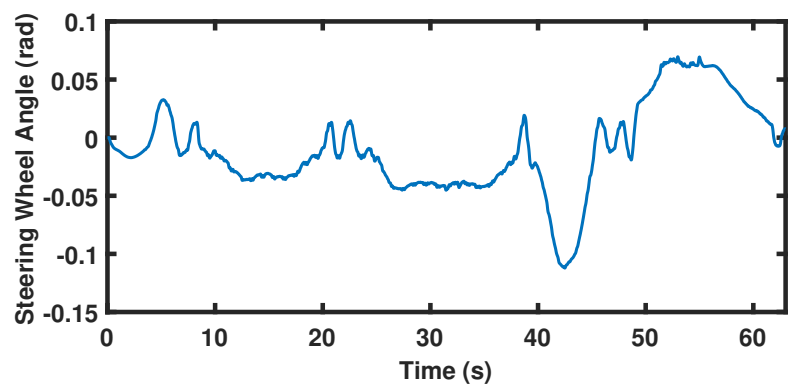

Fig. 10: Steering Wheel Angle (m)

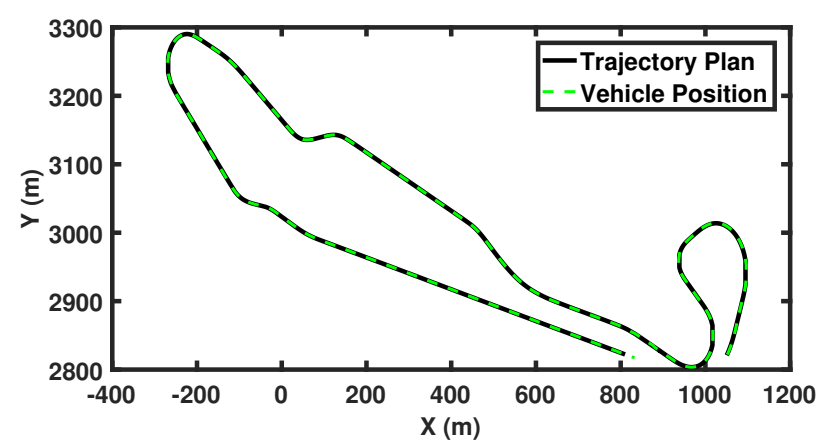

Fig. 11: Satory trajectory plan

road conditions and its inclinations. Moreover, Fig. 12 shows the variation of the longitudinal velocity which is considered as an external parameter of the LPV mode. The velocity evolution is considered to be coherent with respect to the road curvature. It is important to mention that the driver took the driving action between time $\mathrm{t}=95$ and $\mathrm{t}=110$ seconds to re-initialize the path-planning generation (so this interval is out of analysis).

As discussed before, there is a trade-off between exact tracking and passengers' comfortability, and that is why the constraints on the lateral error are relaxed (shown in Fig. 13). As a result, the comfortable riding can be observed in Figs. 14 and 15. Both graphs ensure the actuator limitations represented as:

$$
\left|\dot{\delta}_{\max }\right|=0.2,\left|\delta_{\max }\right|=0.4
$$

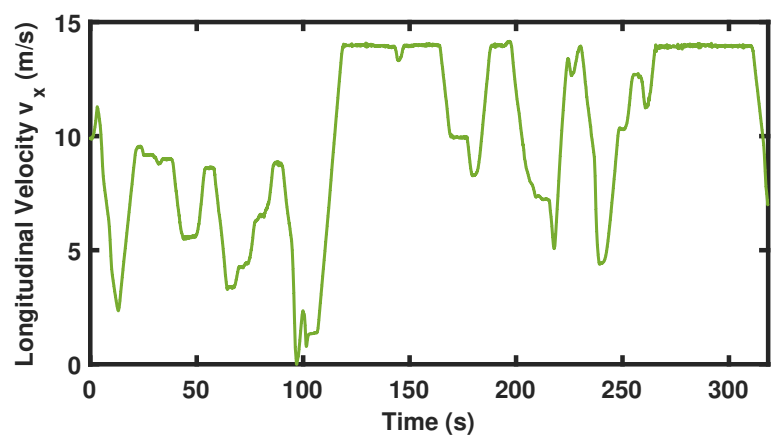

Fig. 12: Longitudinal velocity $v_{x}$ 


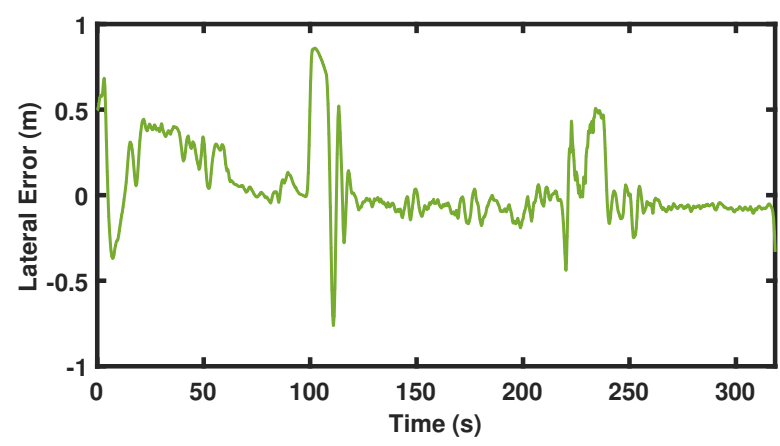

Fig. 13: Lateral error

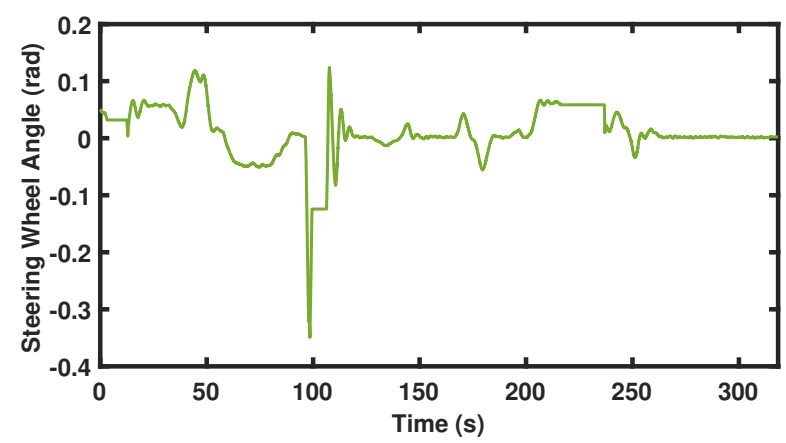

Fig. 14: Steering wheel angle $\delta$

\section{CONCLUSIONS}

In this paper, we have proposed and experimentally validated a new LPV approach for the lateral control of autonomous vehicle. The parameters of the frequency-domain weights used in control design were physically adjusted which facilitates the tuning role. This kind of tuning helps to translate the real actuator limitations to a filter added to the optimisation problem.

The results obtained in real testing were really interesting which opens a new window of applications on LPV approaches in autonomous vehicles. Indeed such a control approach shows the interest to consider system variations and to handle with environmental disturbances (wind, road, etc...).

Future works will concern the improvement of performance tracking simultaneously with actuator limitations in various experimental conditions. A better controller can be designed to attenuate the noises effects found in real tests. Moreover, the LPV gridding approach can be tested and compared to the LFT approach.

\section{ACKNOWLEDGMENT}

Authors express their gratitude to RENAULT research department for its support in developing experimental tests. This paper reflects solely the views of the authors and not necessarily the views of the company they belong to.

\section{REFERENCES}

[1] F. Navas, "Stability analysis for controller switching in autonomous vehicles," Ph.D. dissertation, Sciences Des Metiers De L'Ingenieur (SMI), 2018.

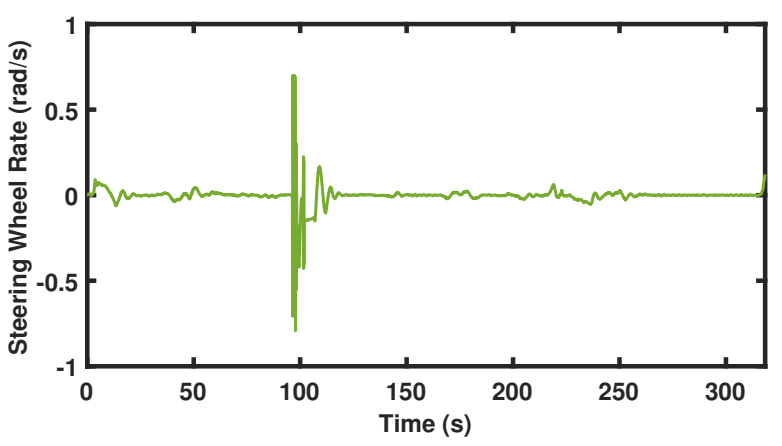

Fig. 15: Steering wheel rate $\dot{\delta}$

[2] H.-S. Tan and J. Huang, "Design of a high-performance automatic steering controller for bus revenue service based on how drivers steer," Robotics, IEEE Transactions on, vol. 30, pp. 1137-1147, 102014.

[3] S. Schnelle, J. Wang, R. J. Jagacinski, and H.-J. Su, "A feedforward and feedback integrated lateral and longitudinal driver model for personalized advanced driver assistance systems," 2018.

[4] R. T. O'Brien, P. A. Iglesias, and T. J. Urban, "Vehicle lateral control for automated highway systems," IEEE Transactions on Control Systems Technology, vol. 4, no. 3, pp. 266-273, 1996.

[5] L. B. Cremean, T. B. Foote, J. H. Gillula, G. H. Hines, D. Kogan, K. L. Kriechbaum, J. C. Lamb, J. Leibs, L. Lindzey, C. E. Rasmussen et al., "Alice: An information-rich autonomous vehicle for high-speed desert navigation," 2006.

[6] S. Chaib, M. S. Netto, and S. Mammar, "H/sub/spl infin//, adaptive, pid and fuzzy control: a comparison of controllers for vehicle lane keeping," in IEEE Intelligent Vehicles Symposium, 2004. IEEE, 2004, pp. 139-144.

[7] S. Sukkarieh, E. Nebot, and H. Durrant-Whyte, "A high integrity imu/gps navigation loop for autonomous land vehicle applications," Robotics and Automation, IEEE Transactions on, vol. 15, pp. 572 578, 071999.

[8] J. Kosecka, R. Blasi, C. Taylor, and J. Malik, "Vision-based lateral control of vehicles," in Proceedings of Conference on Intelligent Transportation Systems. IEEE, 1997, pp. 900-905.

[9] I. Mahtout, F. Navas, D. Gonzalez, V. Milanes, and F. Nashashibi, "Youla-kucera based lateral controller for autonomous vehicle," in 2018 21st International Conference on Intelligent Transportation Systems (ITSC), Nov 2018, pp. 3281-3286.

[10] E. Alcala, V. Puig, J. Quevedo, and T. Escobet, "Gain-scheduling lpv control for autonomous vehicles including friction force estimation and compensation mechanism," IET Control Theory \& Applications, vol. 12, no. 12, pp. 1683-1693, 2018.

[11] E. Alcala, V. Puig, J. Quevedo, and U. Rosolia, "Autonomous racing using linear parameter varying - model predictive control (lpv-mpc)," Control Engineering Practice (under review), vol. 14, no. 8, 2019.

[12] V. Vu, O. Sename, L. Dugard, and P. Gáspár, "The design of an h/lpv active braking control to improve vehicle roll stability." IFAC, 2019.

[13] P. Apkarian and P. Gahinet, "A convex characterization of gainscheduled $H_{\infty}$ controllers," IEEE Transactions on Automatic Control, vol. 40, no. 5, pp. 853-864, May 1995.

[14] E. Roche, O. Sename, and D. Simon, "Lft/hinf varying sampling control for autonomous underwater vehicles," IFAC Symposium on System, Structure and Control, 2010.

[15] C. Gauthier, O. Sename, L. Dugard, and G. Meissonnier, "Some experimental results of an -lpv controller applied to a diesel engine common rail injection system," IFAC Proceedings Volumes, vol. 40, no. 10, pp. 417-424, 2007.

[16] H. Pacejka, Tire and vehicle dynamics. Elsevier, 2005.

[17] R. Rajamani, Vehicle dynamics and control. New York, USA: Springer Science \& Business Media, 2011. [Online]. Available: http://standards.sae.org/j3016201401

[18] H. Kajiwara, P. Apkarian, and P. Gahinet, "Lpv techniques for control of an inverted pendulum," IEEE Control Systems Magazine, vol. 19, no. 1, pp. 44-54, 1999.

[19] J. Mohammadpour and C. W. Scherer, Control of linear parameter 
CONFIDENTIAL. Limited circulation. For review only.

varying systems with applications. Springer Science \& Business Media, 2012.

[20] O. Sename, P. Gaspar, and J. Bokor, Robust control and linear parameter varying approaches: application to vehicle dynamics. Springer, 2013, vol. 437. 